OPEN ACCESS

Edited by: Mikhail Lebedev,

Duke University, USA

Reviewed by:

Christine Portfors,

Washington State University, USA

Donata Oertel,

University of Wisconsin, USA Bopanna Iythichanda Kalappa,

University of Pittsburgh, USA

*Correspondence: Susan E. Shore,

Kresge Hearing Research Institute-Department

of Otolaryngology, University of Michigan, 1150 West Medical Center Drive, Ann Arbor, MI 48109,

USA

sushore@umich.edu

Received: 26 June 2015 Accepted: 30 July 2015 Published: 14 August 2015

Citation:

Wu C, Martel DT and Shore SE (2015)

Transcutaneous induction of stimulus-timing-dependent plasticity in dorsal cochlear nucleus.

Front. Syst. Neurosci. 9:116. doi: 10.3389/fnsys.2015.00116

\section{Transcutaneous induction of stimulus-timing-dependent plasticity in dorsal cochlear nucleus}

\author{
Calvin Wu ${ }^{1}$, David T. Martel ${ }^{1,2}$ and Susan E. Shore ${ }^{1,2,3 *}$ \\ ${ }^{1}$ Kresge Hearing Research Institute-Department of Otolaryngology, University of Michigan, Ann Arbor, MI, USA, \\ ${ }^{2}$ Department of Biomedical Engineering, University of Michigan, Ann Arbor, MI, USA, ${ }^{3}$ Department of Molecular and \\ Integrative Physiology, University of Michigan, Ann Arbor, MI, USA
}

The cochlear nucleus $(\mathrm{CN})$ is the first site of multisensory integration in the ascending auditory pathway. The principal output neurons of the dorsal cochlear nucleus (DCN), fusiform cells, receive somatosensory information relayed by the $\mathrm{CN}$ granule cells from the trigeminal and dorsal column pathways. Integration of somatosensory and auditory inputs results in long-term enhancement or suppression in a stimulus-timing-dependent manner. Here, we demonstrate that stimulus-timing-dependent plasticity (STDP) can be induced in DCN fusiform cells using paired auditory and transcutaneous electrical stimulation of the face and neck to activate trigeminal and dorsal column pathways to the $\mathrm{CN}$, respectively. Long-lasting changes in fusiform cell firing rates persisted for up to $2 \mathrm{~h}$ after this bimodal stimulation, and followed Hebbian or anti-Hebbian rules, depending on tone duration, but not somatosensory stimulation location: 50 ms paired tones evoked predominantly Hebbian, while $10 \mathrm{~ms}$ paired tones evoked predominantly anti-Hebbian plasticity. The tone-duration-dependent STDP was strongly correlated with first inter-spike intervals, implicating intrinsic cellular properties as determinants of STDP. This study demonstrates that transcutaneous stimulation with precise auditorysomatosensory timing parameters can non-invasively induce fusiform cell long-term modulation, which could be harnessed in the future to moderate tinnitus-related hyperactivity in DCN. Keywords: transcutaneous electrical nerve stimulation, stimulus-timing-dependent plasticity, tinnitus, dorsal
cochlear nucleus, multisensory integration

\section{Introduction}

The cochlear nucleus (CN) receives auditory nerve fiber (ANF) inputs from the cochlea, as well as projections from somatosensory afferents. The trigeminal and dorsal column pathways send axonal terminals to the marginal area of the ventral cochlear nucleus (VCN) and the small cell cap, collectively defined as the granule cell domain (GCD; Wright and Ryugo, 1996; Shore et al., 2000; Zhou and Shore, 2004; Zhan et al., 2006; Zeng et al., 2011). CN granule cell axons relay somatosensory inputs to the apical dendrites of fusiform cells and associated inhibitory interneurons (Davis et al., 1996; Davis and Young, 1997; Golding and Oertel, 1997). Electrically stimulating the trigeminal ganglion or the spinal trigeminal nucleus (Sp5), cervical nerve or dorsal column brainstem nuclei can suppress or enhance fusiform cell responses to auditory stimuli (Kanold and Young, 2001; Shore, 2005; Kanold et al., 2011; Koehler et al., 2011; Dehmel et al., 2012). 
Whether enhancement or suppression occurs is determined by the temporal order and interval of the combined auditory and somatosensory stimuli (Koehler and Shore, 2013a,b), which is mediated by spike-timing-dependent plasticity (sTDP) of the parallel fiber-fusiform cell synapse in vitro (Tzounopoulos et al., 2004, 2007). Fusiform cells show "Hebbian plasticity" when presynaptic potentials preceding post-synaptic spikes induce enhancement, while those following spikes induce suppression. Using tone stimulation to generate post-synaptic fusiform cell spikes and somatosensory stimulation to generate pre-synaptic potentials, the macromolecular correlate of sTDP, stimulustiming-dependent plasticity (STDP), can be observed (Koehler and Shore, 2013a,b). STDP is a long-lasting process that likely mediates circuit formation and adaptive filtering of internal vs. external auditory cues (Bell et al., 1997; Nelson, 2004; Oertel and Young, 2004; Requarth and Sawtell, 2011). Maladaptive STDP in DCN occurs in tinnitus (Koehler and Shore, 2013a), the phantom perception of sound, which is characterized by fusiform cell hyperactivity (Brozoski et al., 2002; Kaltenbach et al., 2004, 2005; Dehmel et al., 2012; Koehler and Shore, 2013a).

In this study, we show that STDP can be induced by pairing auditory with transcutaneous activation of somatosensory pathways to the $\mathrm{CN}$. Electrical stimulation of the trigeminal (face) and the dorsal column (neck) afferent pathways produced robust Hebbian or anti-Hebbian plasticity in fusiform cells. STDP was dependent on tone duration and associated with intrinsic properties of fusiform cells. These findings demonstrate a noninvasive approach to control DCN activity, providing feasibility for an effective and accessible tinnitus treatment strategy.

\section{Materials and Methods}

\section{Surgical Preparation and Recording}

All animal procedures were performed in accordance with protocols established by the National Institute of Health publication No. 80-23 and approved by the University Committee on Use and Care of animals at University of Michigan. Guinea pigs ( $n=11$; 326-985 g; Elm Hill Labs) were anesthetized subcutaneously (40 mg/kg ketamine - Putney Inc., $10 \mathrm{mg} / \mathrm{kg}$ xylazine-Lloyd Inc.) and secured with hollow ear bars in a stereotaxic frame (Kopf). Body temperature was kept constant at $38^{\circ} \mathrm{C}$ with a custom built encapsulating thermal pad and rectal probe. Anesthetic depth was assessed by hind leg withdrawal areflexia and maintained with hourly $10 \mathrm{mg} / \mathrm{kg}$ ketamine and $3 \mathrm{mg} / \mathrm{kg}$ xylazine injections. Incision sites were treated with topical lidocaine. Recordings were performed in a double walled sound-proof booth. Auditory brainstem responses were assessed prior to surgery to establish normal hearing thresholds. After a small craniotomy, a two-shank 16-channel recording probe (NeuroNexus) was placed stereotaxically into DCN through an intact cerebellum at a $25^{\circ}$ angle from vertical, $4 \mathrm{~mm}$ caudal of interaural line, $3 \mathrm{~mm}$ lateral of midline, and a depth of 6-7 mm. Broadband noise bursts (65 dB SPL, $50 \mathrm{~ms}$ duration, $2 \mathrm{~ms}$ linear ramp rise/fall time) were used to locate units. Receptive fields $(100-24 \mathrm{kHz}$ tone bursts in 0.15 octave steps; 0-90 dB in $5 \mathrm{~dB}$ steps; 50 ms duration, 2 ms linear ramp rise/fall time) were recorded to determine thresholds and best frequencies (BFs). A suitable electrode location in the DCN fusiform cell layer was confirmed by robust responses to BF tones with buildup or pauser-buildup temporal patterns and type III tuning properties that are typical of DCN fusiform cells (Stabler et al., 1996). Animals were terminated with sodium pentobarbital at the end of each experiment.

\section{Transcutaneous Electrical Stimulation}

Animals were shaved in regions of transcutaneous electrode placement: for trigeminal pathway stimulation, an electrode pad (10 mm diameter Ag/AgCl Brainet electrode, Rhythmlink) was placed on the skin at the center of the left cheek superficial to the masseter muscle, with the ground electrode pad on the nasal bridge (Figure 1A). For dorsal column pathway stimulation, the electrode pad was placed on the neck $1 \mathrm{~cm}$ caudal to the ridge of the occipital bone and $1 \mathrm{~cm}$ lateral (left) to the midline. A ground electrode was placed $1 \mathrm{~cm}$ medial to the active electrode (Figure 1A). Three biphasic (100 $\mu \mathrm{s} /$ phase) current pulses at $1000 \mathrm{~Hz}$ (2.2 ms total duration) were delivered per trial at levels that evoked fusiform cell spikes above spontaneous rate $(2-5 \mathrm{~mA})$.

\section{Assessment of STDP}

To assess STDP, $40 \mathrm{~dB}$ spike latency (SL) tones at BF $(50 \mathrm{~ms}$ duration, $2 \mathrm{~ms}$ linear ramp rise/fall time, 100 repetitions at 5 per second) were presented before bimodal (auditorysomatosensory) pairing. 300 repetitions ( 5 per second) of either $50 \mathrm{~ms}$ or $10 \mathrm{~ms}$ duration tones ( $2 \mathrm{~ms}$ linear ramp rise/fall time) were paired with transcutaneous electrical stimulation of either trigeminal or dorsal column pathways with different bimodal intervals (temporal gap of electrical pulses relative to the tones). Bimodal intervals were either positive (electrical-preceding) or negative (auditory-preceding; Figure 1B). Responses to bimodal stimuli were compared to responses to the $40 \mathrm{~dB}$ SL BF tones alone, presented before, 5 and $15 \mathrm{~min}$ after bimodal stimulation to assess long-term changes in fusiform cell firing rates. In some experiments, persistent effects were assessed for up to 60 or $120 \mathrm{~min}$ to determine recovery. Persistent effects were quantified as percent changes in firing rate from the control, prebimodal tone alone condition. Recovery was defined as return to within $\pm 10 \%$ of the pre-bimodal baseline at the earliest assessment time point after maximum bimodal enhancement or suppression. As a control, bimodal pairing was replaced with unimodal auditory or unimodal electrical stimulation. Bimodal intervals $(-20,-10,-5,5,10,20 \mathrm{~ms})$, and unimodal auditory and electrical stimulation were randomized in each experiment. In some experiments, only one bimodal interval was used to control for potential effects of repeated stimuli.

\section{Data Analysis}

Voltages recorded from multi-channel recording probes were digitized by a PZ2 (Tucker Davis Technologies) preamplifier and band-pass filtered (300-3 kHz) for spike detection: threshold was set at 2.5 SD above background noise. Recorded spike waveforms were sorted by principal components of the waveform shape and cluster analyses (Plexon Offline Sorter). Electrical 


\section{A}

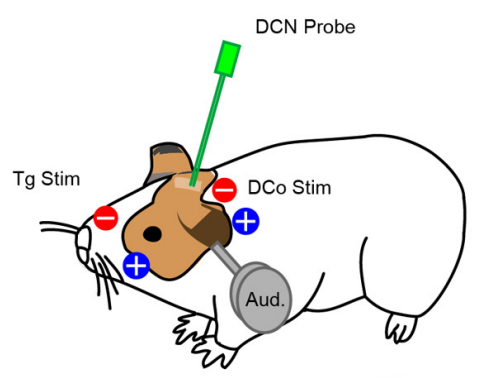

FIGURE 1 | Transcutaneous electrical stimulation and bimodal pairing protocol. (A) Skin electrode pads were placed on the ipsilateral face and neck to activate the trigeminal (Tg) and dorsal column (DCo) somatosensory pathways. DCN neural responses were recorded during electrical and auditory

B

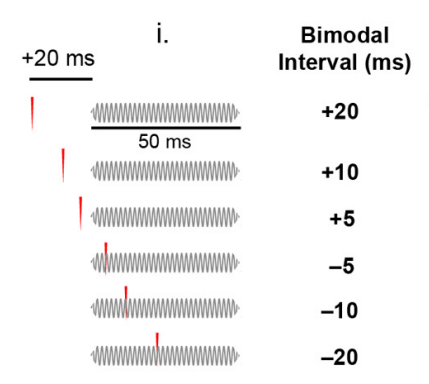

ii.

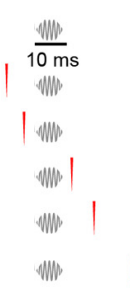

stimulation (Aud). (B) Bimodal pairing using $50 \mathrm{~ms}$ (i) or $10 \mathrm{~ms}$ (ii) tones in a close temporal proximity with transcutaneous electrical stimulation. Positive bimodal intervals indicate preceding electrical stimulation while negative intervals indicate preceding auditory stimulation. artifacts were identified by their distinct clusters and removed from further analysis. Spike waveforms remained consistent over the recording duration. Sorted spikes were imported to MATLAB as timestamps. Spike latencies were calculated using the bin-less Poisson method described by Chase and Young (2007). STDPs were presented as percent changes in spike rate as a function of bimodal interval, and classified into Hebbian or anti-Hebbian types. Other types of STDP such as enhancement or suppression reported previously in vivo (Koehler and Shore, 2013b) were not considered in further analysis. STDP indices (sums of relative changes for positive bimodal intervals subtracted from sums of relative changes for negative intervals) were computed to quantify Hebbian or antiHebbian tendencies. Statistical analyses were performed using the MATLAB statistical toolbox. Two-way analysis of variance (ANOVA), as well as non-parametric statistical tests for unequal population distributions were used. Post hoc tests for ANOVAs were conducted using the Tukey-Kramer method. Significance was established at $\alpha=0.05$.

\section{Results}

\section{Transcutaneous Trigeminal and Dorsal Column Somatosensory Stimulation Activate Distinct Pathways to DCN}

Both face (trigeminal) and neck (dorsal column) unimodal electrical stimulation evoked responses in identified fusiform cells similar to those previously reported using deep brain stimulation (Koehler and Shore, 2013a,b). 19 of 155 (12\%) units responded to face stimulation and 43 of 129 (33\%) units responded to neck stimulation. Figure 2A shows a representative unit response to unimodal electrical stimulation of the face: a sharp onset response followed by weak sustained firing. Evoked firing rates (averaged over $20 \mathrm{~ms}$ after the onset response) were similar for facial and neck stimulation (Mann-Whitney $U=573, P=0.12$; Figure 2B). The modes of the first spike latency (FSL) distributions were 3 and $11 \mathrm{~ms}$ for face stimulation and $4-5 \mathrm{~ms}$ for neck stimulation (Figure 2C). The median FSLs were 12 and $5 \mathrm{~ms}$, respectively (Figure 2C, inset). The difference in FSL distributions was significant (KolmogorovSmirnov test, $Z=0.38, P=0.041$ ), suggesting that these inputs were transmitted to the DCN via separate (trigeminal vs. dorsal column) pathways (Wright and Ryugo, 1996; Zhou and Shore, 2004; Haenggeli et al., 2005; Zeng et al., 2011).

\section{Paired Auditory-Somatosensory Stimulation Induces Long-term Enhancement or Suppression of Fusiform Cell Responses}

Studies using paired auditory and deep brain spinal trigeminal nucleus (Sp5) stimulation demonstrated long-lasting in vivo plasticity in fusiform cells (Dehmel et al., 2012; Koehler and Shore, 2013a,b). Plasticity induction required bimodal pairings comprised of repeated presentations of Sp5 and auditory stimuli with short temporal gaps. In the present study, Sp5 stimulation was replaced with transcutaneous activation of the trigeminal pathways. In addition, the dorsal column pathways were activated via neck stimulation. Figure 3A shows an example of plasticity induction. The fusiform cell response to tones $15 \mathrm{~min}$ after bimodal pairing was compared with the control (response to the tone alone) before bimodal pairing. In this figure, a temporal gap of $10 \mathrm{~ms}$ ("bimodal interval": $\mathrm{BI}=+10 \mathrm{~ms}$ ) produced immediate enhancement. Enhancement, or suppression in other cases, could persist for more than $120 \mathrm{~min}$ (Figure 3B). Maximum effects were observed at a median of $60 \mathrm{~min}$, and recovery from plasticity at $90 \mathrm{~min}$. Bimodal pairing produced greater enhancement and suppression than repeated tones or electrical stimulation alone (Figures 3C-E).

\section{Hebbian or anti-Hebbian Plasticity is Dependent on Pairing Tone Duration}

Whether fusiform cells show enhancement or suppression is dependent on the BI (Koehler and Shore, 2013a,b). In addition to varying the BI as in Koehler and Shore (2013a,b), two more variables were introduced here: stimulus location and pairing tone (PT) length (Figure 1B). Figures 3F-I display representative STDP for face (trigeminal) and neck (dorsal column) stimulation with PT durations of 10 and $50 \mathrm{~ms}$. STDP indices were anti-Hebbian for units in Figures 3F,H, and Hebbian 


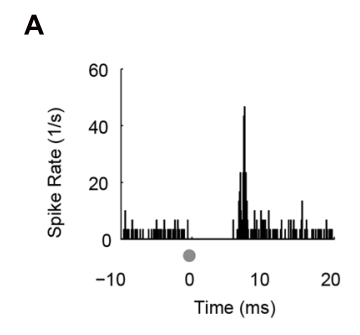

B

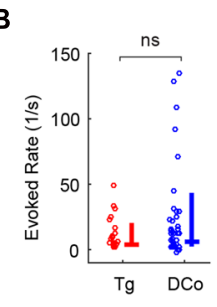

C

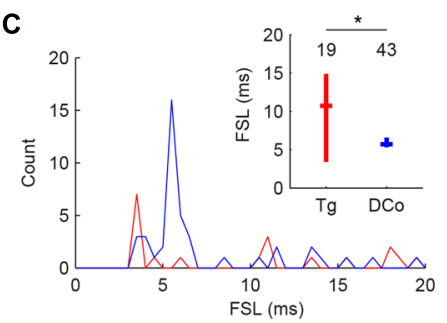

FIGURE 2 | Transcutaneous somatosensory stimulation activates separate pathways to DCN. (A) Representative temporal responses of fusiform cells to transcutaneous electrical stimulation (gray dots at time 0). The absence of spikes before the onset is due to artifact removal during spike sorting. Onset latencies, rates and subsequent sustained responses (Sus) were quantified in (B,C). Unimodal face/trigeminal (Tg; red) and neck/dorsal column (DCo; blue) stimulation evoked similar onset (B) and sustained responses (C), but showed different first-spike latency (FSL) distributions (inset). Inset in (C): median and quartile FSL; values above the plots represent the number of units. $* P<0.05$. for units in Figures 3G,I. The population STDP corresponding to pairing schemes of Figures 3F-I is shown in Figures 4A-D. Experiments in which only one BI was assessed (see circles in Figures 4A,C,D) demonstrated that interleaved bimodal pairing protocol did not affect subsequent plasticity $(P>0.05$ for one-BI vs. BI randomized). Face/trigeminal stimulation induced predominantly anti-Hebbian plasticity with $10 \mathrm{~ms}$ PT [KruskalWallis test, $\left.H_{(5)}=18.6, P=0.0023\right]$ and Hebbian plasticity with $50 \mathrm{~ms}$ PTs $\left[H_{(5)}=18.5, P=0.0024\right]$. Neck/dorsal column stimulation also induced both anti-Hebbian and Hebbian plasticity that was determined by PT duration: anti-Hebbian with $10 \mathrm{~ms} \mathrm{PT}\left[H_{(5)}=26.2, P=8.1 \times 10^{-5}\right]$ and Hebbian with $50 \mathrm{~ms}$ PT $\left[H_{(3)}=16.1, P=0.0066\right]$.

To quantify population tendencies for Hebbian or antiHebbian plasticity, the sum of relative changes for the positive intervals $(+5,+10$, and +20 BIs) was subtracted from the sum of relative changes for the negative intervals $(-5,-10$, and -10 BIs) resulting in a Hebbian timing rule with a positive index and an anti-Hebbian timing rule with a negative index (Figure 4E). A larger difference with PT duration was observed for neck/dorsal column stimulation. Median and quartile STDP indices for the four different pairing schemes are plotted in Figure 4E. A post hoc analysis of ANOVA (Kruskal-Wallis test, $H_{(3)}=14.3, P=0.003$ ) showed a significant difference between 10 and $50 \mathrm{~ms}$ PTs for neck/dorsal column stimulation but not for face/trigeminal stimulation.

Differences in the effects of PT duration can also be observed by counting the proportion of STDP rules produced using either the 50 or $10 \mathrm{~ms}$ duration (Figure 4F). For both face/trigeminal and neck/dorsal column stimulation, $10 \mathrm{~ms}$ PTs produced predominantly anti-Hebbian timing rules while $50 \mathrm{~ms}$ PT produced predominantly Hebbian timing rules. STDP proportions were significantly different between PT duration $\left(\chi_{(3)}^{2}=10.5, P=0.014\right.$ for trigeminal and $\chi_{(3)}^{2}=26.2$, $P=8.6 \times 10^{-6}$ for dorsal column) but not stimulation location $\left(\chi_{(3)}^{2}\right)=7.7, P=0.052$ for $10 \mathrm{~ms}$ PT and $\chi_{(3)}^{2}=2.9, P=0.41$ for $50 \mathrm{~ms}$ PT). To confirm the effect of PT duration, STDP curves were generated in the same units using both 10 and $50 \mathrm{~ms}$ PT paired with face/trigeminal stimulation (Figure 5A). In this instance, BIs for $10 \mathrm{~ms}$ PTs and $50 \mathrm{~ms}$ PTs were randomized (both PTs, interleaved with all BIs). 15 out of 23 units showed
STDP reversals from anti-Hebbian to Hebbian when PT was changed from 10 to $50 \mathrm{~ms}$ (Figure 5B). The mean STDP index increased, as expected (Wilcoxon's signed-rank test, $Z=-3.3$, $\left.\mathrm{P}=8.2 \times 10^{-4}\right)$.

\section{Fusiform Cell Tonal Responses Predict STDP Outcome}

Since STDPs are largely influenced by PT duration, mechanisms that control STDP may be modulated differently by 10 or $50 \mathrm{~ms}$ tones. First, $50 \mathrm{~ms}$ tones produced higher spike counts than $10 \mathrm{~ms}$ tones $\left(U=11,708, P=8.2 \times 10^{-4}\right)$. However, spike rate did not correlate with STDP outcomes [Figure 6A; Kendall's tau, $\left.r_{(123)}^{2}=-0.04, P=0.67\right]$, suggesting that the amount of tone-evoked spikes during bimodal pairing does not affect STDP. Instead, $50 \mathrm{~ms}$ tones activated fusiform cells for a longer time (median duration with significant evoked spikes: $55.4 \mathrm{~ms}$ ) than $10 \mathrm{~ms}$ tones (median: $15.7 \mathrm{~ms}$ ). The duration of evoked activities, or interval with significant PTevoked responses, indeed correlated with timing rule outcomes $\left[r_{(139)}^{2}=0.31, P=1.4 \times 10^{-4}\right]$. The difference in timing rule outcome may, therefore, be due to the duration of sustained firing: fusiform cells typically show pauser or buildup temporal patterns (Figure 6B, upper panel), and $10 \mathrm{~ms}$ tones are not long enough to generate buildup phases, in which delayed first spikes or second spikes occur. Thus, we hypothesized that the buildup phases may determine STDP outcomes. To quantify the buildup phase, first inter-spike intervals (FISI) were calculated (Figure 6B, lower panel); a higher FISI value indicated a longer buildup phase. A significant correlation was found between the mean FISI (as a percentage of total duration of evoked activity) of each fusiform cell and STDP index $\left[r_{(85)}^{2}=-0.43, P=3.5 \times 10^{-5}\right.$; Figure 6C]. STDP became anti-Hebbian as shorter tones increased the relative FISIs. To rule out possible effects of bimodal plasticity on FISI, we showed that FISI remained stable throughout the experimental duration [Kruskal-Wallis test, $H_{(7)}=7.5, P=0.38$; Figure 6D].

The buildup temporal pattern of fusiform cells is also influenced by inhibition (Kanold and Manis, 1999, 2001, 2005), likely derived from vertical cells in the deep layer of DCN (Rhode, 1999; Muniak and Ryugo, 2014). Thus, we tested 
A

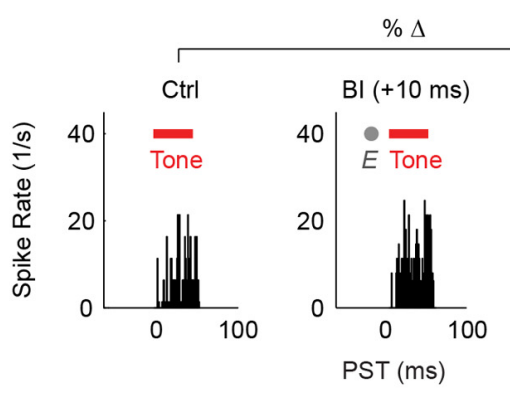

C

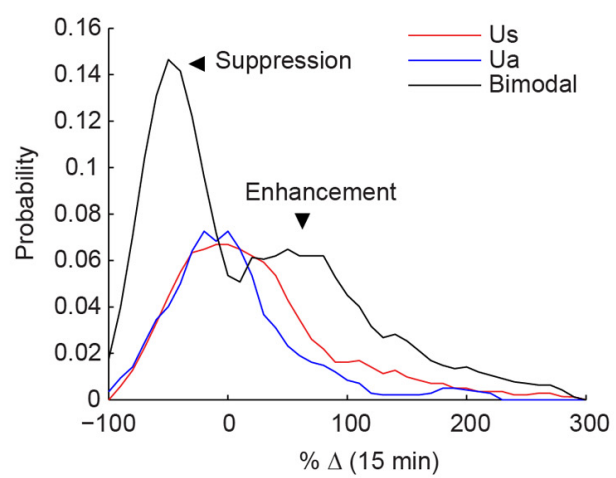

D

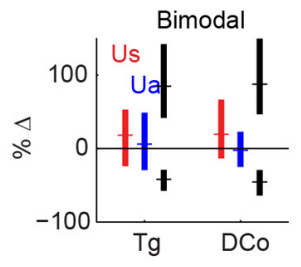

E

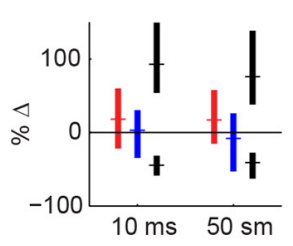

FIGURE 3 | Stimulus-timing-dependent plasticity induced by paired auditory and transcutaneous somatosensory stimulation. (A) An example of long-lasting enhancement (15 min) induced by paired face $/ \mathrm{Tg}$ transcutaneous and $50 \mathrm{~ms}$ tone stimulation at a bimodal interval $(\mathrm{Bl})$ of $+10 \mathrm{~ms}$. Plasticity is quantified as percent change in tone-evoked activity from pre- (control; Ctrl) to post-bimodal stimulation. Tone duration is denoted by red bars and electrical stimulation (E) by the gray dot. (B) Changes in tone-evoked activity from control to 0 (during pairing), 5, 15, 30, 60, 90, and 120 min after bimodal pairing.

(C) Distribution (histogram) of percent change in tone-evoked responses from
B

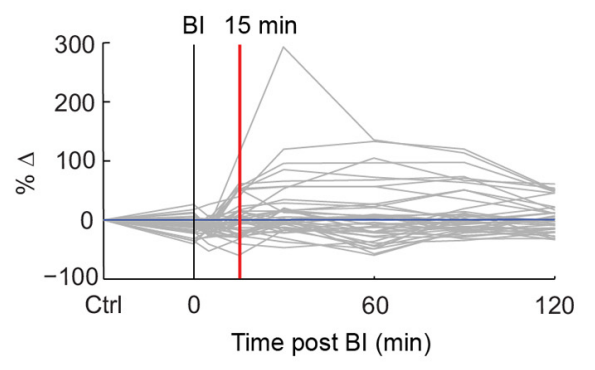

G
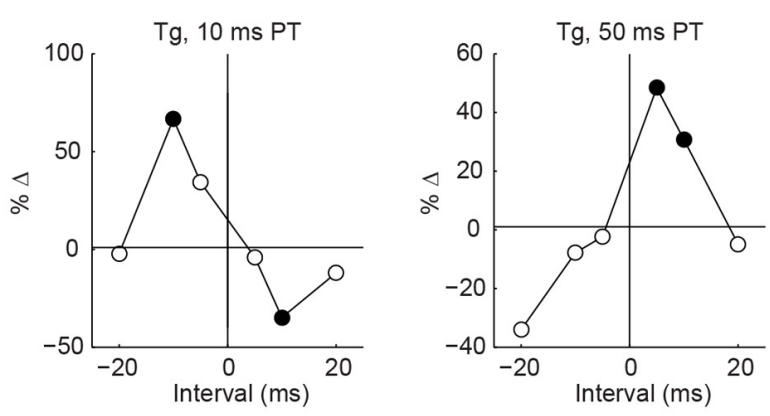

H
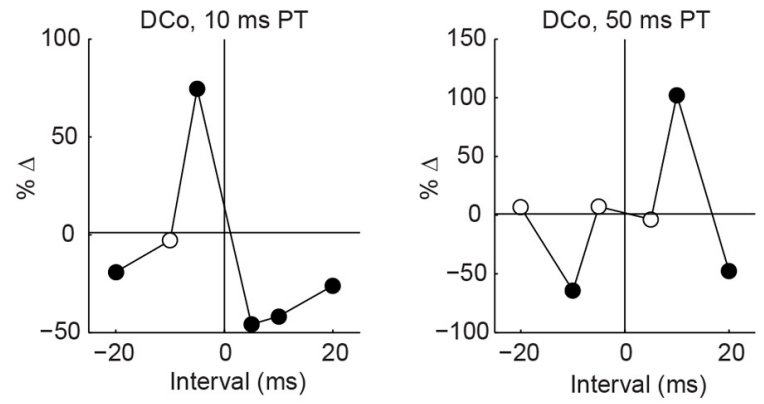

control to 15 min post-bimodal pairing. Unimodal auditory (Ua) and somatosensory (Us) stimulation produce median changes of 0 , while bimodal stimulation produces enhancement (positive change) or suppression (negative change). (D,E) Median and quartile percent changes parsed by activation pathway: (D) face/ Tg or neck/dorsal column (DCo); (E) or pairing tone (PT) duration (10 or 50 ms). (F-I) Percent changes as functions of bimodal intervals, or "timing rules", for different stimulus parameters: (F) face/Tg with $10 \mathrm{~ms}$ PTs or (G) $50 \mathrm{~ms}$ PT, (H) neck/DCo with $10 \mathrm{~ms}$, or (I) $50 \mathrm{~ms}$ PT. Closed circles, significant changes from 0 ; open circles, no significance. whether units receiving stronger inhibition produce different STDP. Inhibition was identified by the degree of monotonicity in rate-level functions and categorized into four types: inhibition to below spontaneous rate $(-2)$, highly non-monotonic $(-1)$, weakly non-monotonic (0), and monotonic (1) (Figure 7A). Monotonic units showed slight trends toward higher STDP indices, or more Hebbian timing rules (Figure 7B); however, this was not statistically significant. Nevertheless, the difference between the 10 and the $50 \mathrm{~ms}$ PT-induced STDP was greater in non-monotonic units [two-way ANOVA, $F_{(2,1)}=5.4$, $P=0.02$ for PT duration, $P<0.05$ for -1 and 0 degree monotonicity], confirming a role of inhibitory influence in bimodal plasticity.

\section{Discussion}

In this study we used transcutaneous electrical stimulation to activate trigeminal and dorsal column somatosensory afferent pathways to the DCN. The response properties evoked in this manner were consistent with those described with deep brain stimulation. First, the response latencies of fusiform cells in 
A

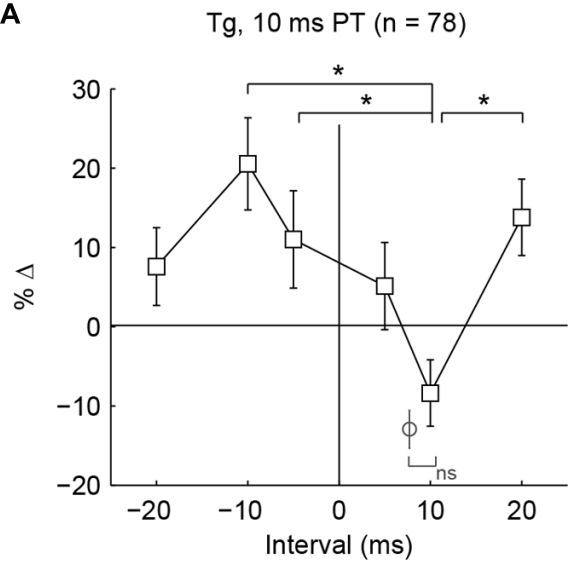

C

DCo, $10 \mathrm{~ms}$ PT $(n=55)$

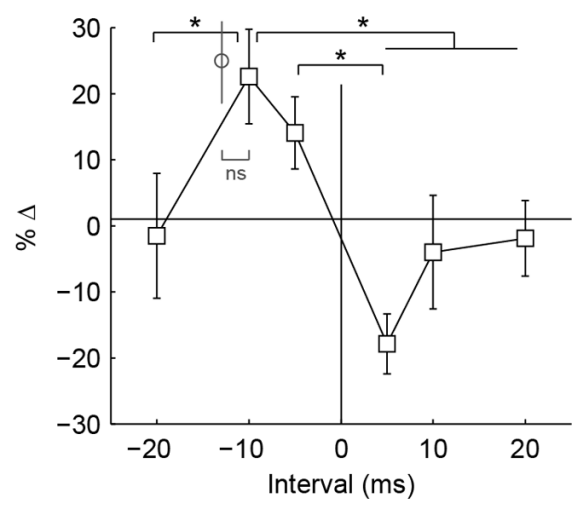

E

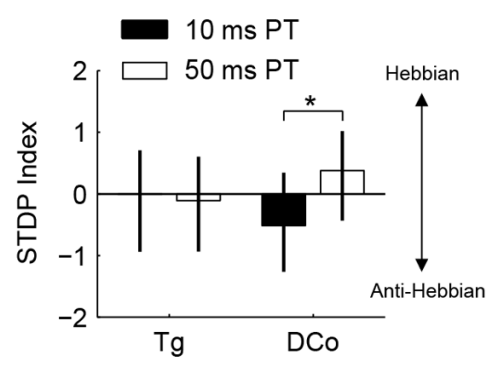

FIGURE 4 | Whether population timing rules are Hebbian or anti-Hebbian depends on PT duration. (A-D) Percent changes in mean tone-evoked rates as functions of bimodal intervals (timing rules) corresponding to Figures $\mathbf{3 C - F}$. Additional data points (gray circles) outside of line plots were collected in control experiments wherein a single bimodal interval was used in one experiment (ns: no significance; ${ }^{*} P<0.05$ )
B

Tg, 50 ms PT $(n=77)$

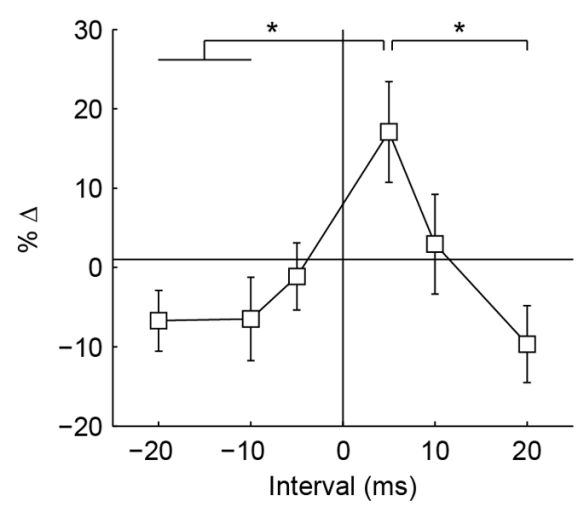

D

$\mathrm{DCo}, 50 \mathrm{~ms}$ PT $(\mathrm{n}=74)$

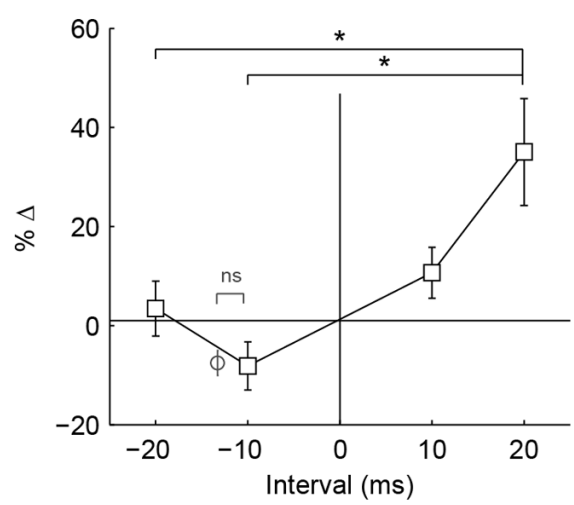

$\mathbf{F}$

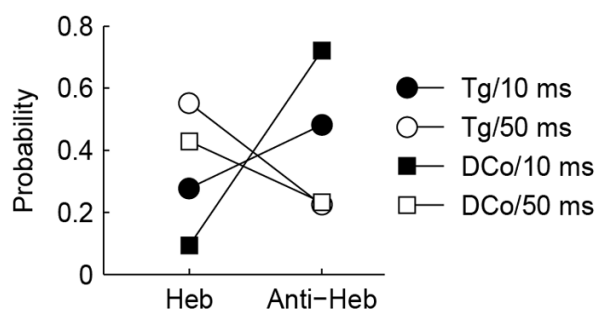

(E) Median and quartile STDP index for face/ Tg and neck/ DCo using 10 or 50 ms duration PTs. Index represents the difference between sums of relative changes for positive and negative bimodal intervals. A positive index indicates a Hebbian-like timing rule while a negative index indicates an anti-Hebbian-like timing rule. (F) Distribution of Hebbian (Heb) and anti-Hebbian (Anti-Heb) rules by stimulus parameters. response to face and neck stimulation were consistent with those to trigeminal ganglion (Shore et al., 2003) and cervical nerve stimulation (Kanold and Young, 2001), respectively. Both types of transcutaneous electrical stimulation produced similar, complex responses in fusiform cells (mixed excitation and inhibition; Davis and Young, 1997; Shore, 2005), suggesting activation of the shared granule cell circuit in DCN. Granule cells extend parallel fiber axons into the DCN molecular layer and synapse on the apical dendrites of fusiform and the inhibitory interneurons, cartwheel cells (Mugnaini et al., 1980; Smith and Rhode, 1985), where STDP occurs. Fusiform cells show Hebbian plasticity, while cartwheel cells show anti-Hebbian plasticity in vitro (Tzounopoulos et al., 2004). In the complex in vivo environment, however, STDP patterns across the fusiform cell population were not uniform (Koehler and Shore, 2013b). In addition, the present study showed that changing stimulus parameters can play a 
A

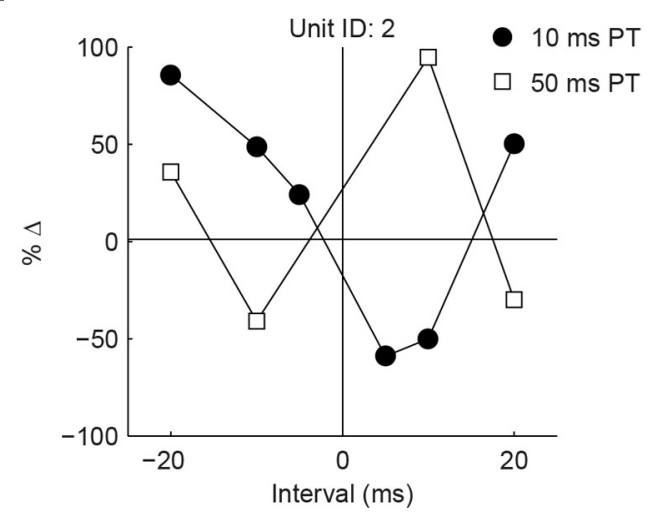

B

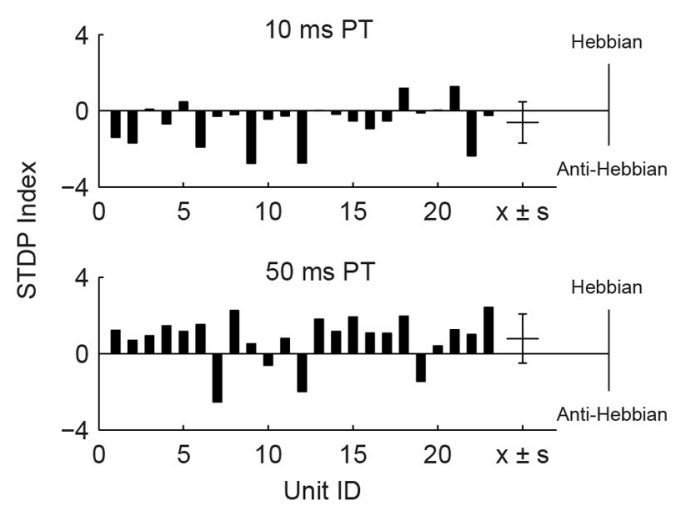

FIGURE 5 | Hebbian or anti-Hebbian timing rules can be induced in the same units using different duration PTs. (A) Timing rules induced by neck/dorsal column stimulation paired with 10 and 50 ms PTs in a representative unit. (B) STDP indices of 23 units using 10 ms PT (upper panel) or 50 ms PT (lower panel). Mean \pm SD $(x \pm s)$ STDP indices shown on the right.

\section{A}

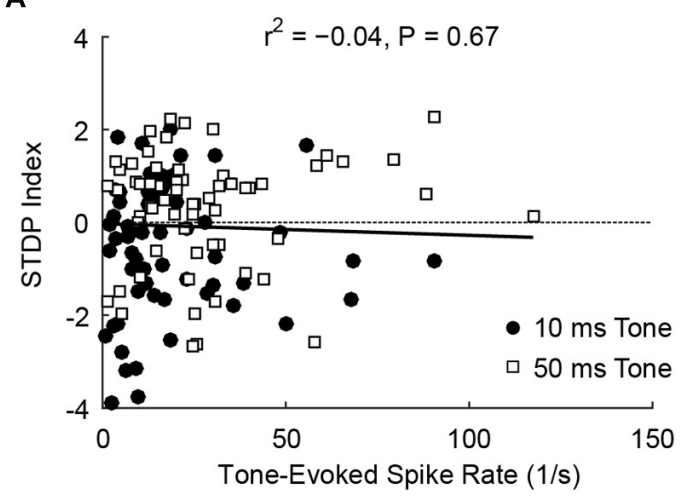

C

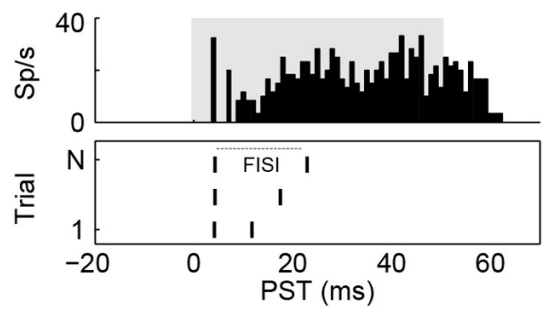

FIGURE 6 | STDP is determined by first inter-spike intervals of tone-evoked responses. (A) STDP index does not correlate with tone-evoked spike rate. Tonal responses (10 and $50 \mathrm{~ms}$ ) were evaluated before STDP assessment. (B) An example for calculating FISI in a pauser unit (upper panel; $50 \mathrm{~ms}$ tones at BF and $20 \mathrm{~dB} \mathrm{SL}$ ). The raster plot (lower panel) shows first and second spikes across trials $(N)$. (C) Mean FISI as a percentage of total evoked
B

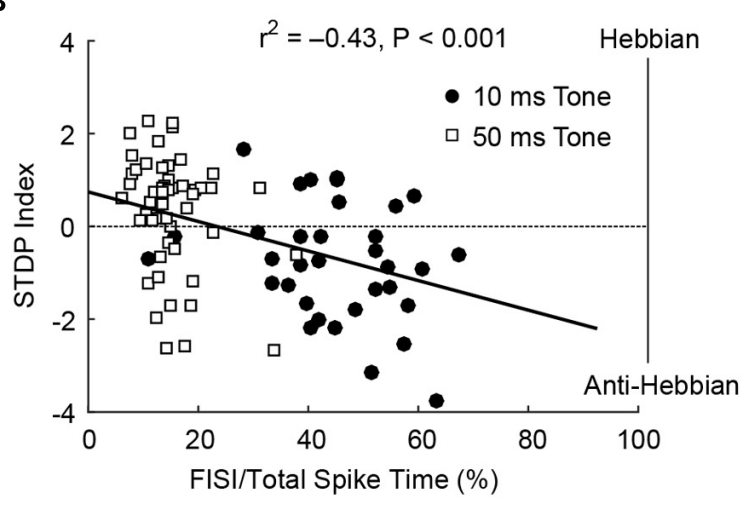

D

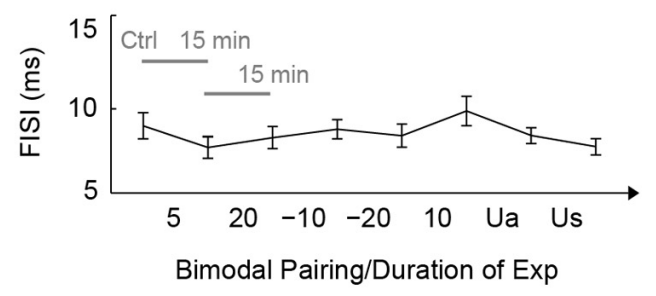

spike activity correlates with STDP index. (D) FISIs were tracked across the duration of an STDP experiment ( $n=30$ units, paired $50 \mathrm{~ms}$ tones and face/Tg stimulation). The first data point was assessed before STDP induction, subsequent data points were assessed 15 min after each bimodal interval. The bimodal pairing protocol used is indicated between each data point. $\mathrm{Ua}$, unimodal auditory; Us, unimodal somatosensory. role in the resulting plasticity, even in the same fusiform cells. Replacing Sp5 (Koehler and Shore, 2013b) with transcutaneous facial stimulation while persevering other parameters, replicated the previous results. Stimulating a different somatosensory afferent pathway preserved the observed plasticity outcomes, while changing PT duration resulted in a consistent change in the plasticity patterns. It is interesting that the differential effect of PT duration is more apparent for neck/dorsal column stimulation, alluding to functional differences between input pathways.

\section{Why Does Changing PT Duration Alter STDP Plasticity Patterns?}

Stimulus-timing-dependent plasticity specificity between fusiform cells and cartwheel cells in vitro is mediated by 
A
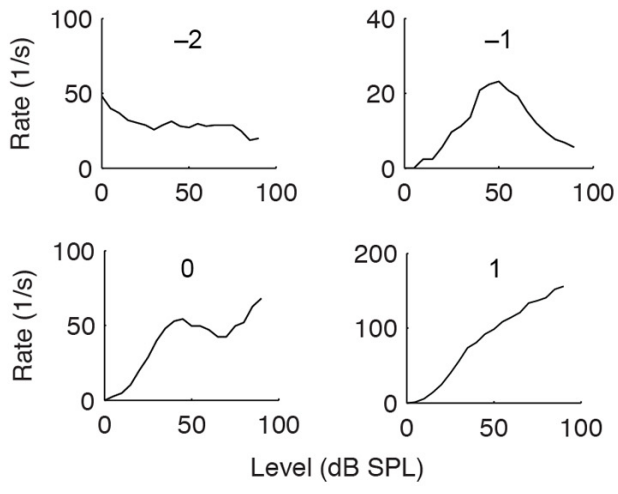

FIGURE 7 | Stronger inhibition predicts Hebbian-anti-Hebbian timing rule divergence. (A) Four representative units' rate-level responses showing different degrees of monotonicity. Monotonicity of -2 indicates highly

non-monotonic responses (strong inhibition) while that of 1 indicates monotonic

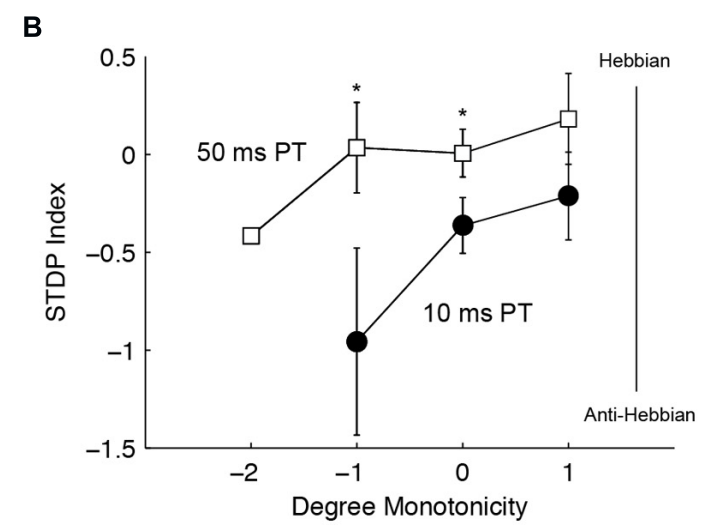

responses (weaker or no inhibition). (B) STDP index as a function of monotonicity. Non-monotonic (degree $-2,-1,0$ ) and monotonic (degree 1) units that underwent both STDP induction using 10 or 50 ms PT were compared. $* P<0.05$. input-selective endocannabinoid signaling (Tzounopoulos et al., 2007; Sedlacek et al., 2011) and cholinergic modulation of the parallel fiber synapses (Zhao and Tzounopoulos, 2011). These factors likely underlie fusiform cell STDP heterogeneity in vivo. However, if STDP inversion from Hebbian to anti Hebbian can be induced by changing PT duration in the same cell, there must be an additional mechanism present either at the ANF-fusiform cell synapse or fusiform soma that contributes to diverse STDP patterns in vivo.

A possible mechanism was suggested by the predictive relationship between fusiform cell temporal patterns and STDP outcomes. The fast-inactivating, A-type potassium channels produce the characteristic pauser/buildup temporal patterns in fusiform cells as a result of delayed spiking after prolonged hyperpolarization (Manis, 1990; Kanold and Manis, 1999). A-type potassium channels also regulate signal transduction and excitability in dendrites of hippocampal pyramidal cells (Johnston et al., 2003; Frick et al., 2004), thereby likely affecting spike timing. Thus, the correlation between fusiform cell temporal responses and STDP implicates the A-type potassium channel as an underlying factor. This hypothesis also explains the observation that cells receiving stronger inhibitory influences (hyperpolarization) also show more anti-Hebbian STDP, consistent with previous findings (Koehler and Shore, 2013b). Thus, differences in intrinsic properties among fusiform cells may result in STDP heterogeneity and PT-dependent alteration. However, a key question yet to be answered is how differential modulation of A-type potassium channels affects STDP.

Alternatively, it may be that PT duration exerts differential effects on the ANF-fusiform cell synapse. This is less likely as ANF synapses on fusiform cells do not show long-term plasticity (Fujino and Oertel, 2003). However, a recent study reported that vertical cell inhibition of fusiform cells can undergo short-term facilitation (Sedlacek and Brenowitz, 2014), which can adjust the fusiform cell output. During the pairing protocol for STDP induction, repeated PT stimulation (of different duration tones) may be modulated by different short-term effects; short-term synaptic modifications can also influence long-term plasticity (Harvey-Girard et al., 2010).

\section{Functional Implications}

Hebbian and anti-Hebbian timing underlie different neural processes. The electrosensory system of the mormyrid electric fish serves as an example of anti-Hebbian functionality (Bell, 1982; Roberts and Leen, 2010). The animal produces electrical discharges for communication, while also relying on electrosensation for movement and navigation. To distinguish between internally generated electric fields from environmental cues, the electrosensory lobe (ELL; a structural analog of the mammalian cerebellum and DCN) receives a copy of the electrical discharge command. ELL neural activity is suppressed when the command signals (feedback) arrive prior to sensory input; sensory input that precedes command feedback is otherwise amplified. This gain adjustment via an anti-Hebbian mechanism is also evident in the mammalian cerebellum (Piochon et al., 2012). Hebbian plasticity, on the other hand, sensitizes rather than suppresses corollary stimuli. This process is important for the development of neural circuits, such as experience-dependent formation of visual fields in the optic tectum (Mu and Poo, 2006), visual cortex (Yao and Dan, 2001), or refinement of the auditory cortical tuning map (Dahmen et al., 2008). In the context of bimodal integration in DCN, anti-Hebbian plasticity likely modulates adaptive filtering. For instance, mastication produces internally generated sounds as well as orofacial inputs (via the trigeminal pathway) to $\mathrm{DCN}$. When the internal feedback signal precedes auditory input, fusiform cell output is suppressed via anti-Hebbian plasticity, and perception of internally generated sound is attenuated. The dorsal column pathway, on the other hand, transmits information regarding neck motion, which changes the head-related transfer function that is detected in DCN (Oertel and Young, 2004). It is likely that internally generated alterations in sound localization cues are also suppressed via anti-Hebbian plasticity. Interestingly, if the generated sounds persist for a longer duration, the circuit 
would shift toward Hebbian plasticity, perhaps as a mechanism to "unlearn" or "reset" an adaptive filter and adjust circuit connectivity.

\section{Induction of STDP: Toward Undoing Pathological Circuitry in Tinnitus}

Tinnitus, the phantom auditory perception, is correlated with fusiform cell hyperactivity (Brozoski et al., 2002; Kaltenbach et al., 2004; Roberts et al., 2010; Dehmel et al., 2012; Koehler and Shore, 2013a; Stefanescu et al., 2015). In addition, tinnitus changes auditory-somatosensory plasticity: using the same stimulus parameters (50 ms PT), normal animals showed predominant Hebbian STDP while tinnitus animals showed anti-Hebbian STDP (Koehler and Shore, 2013a). The Koehler and Shore study suggested that STDP was involved in tinnitus pathophysiology, and that fusiform cell hyperactivity is correlated with altered STDP. Thus, targeting STDP can provide a key to tinnitus treatment. In this study, we demonstrated feasibility for such a bimodal treatment strategy. STDP can be induced by applying transcutaneous electrical stimulation with tone-pairing with specific durations and bimodal intervals. Non-invasive, longterm modulation of fusiform cell activity may provide remedies

\section{References}

Bell, C. C. (1982). Properties of a modifiable efference copy in an electric fish. J. Neurophysiol. 47, 1043-1056.

Bell, C. C., Han, V. Z., Sugawara, Y., and Grant, K. (1997). Synaptic plasticity in a cerebellum-like structure depends on temporal order. Nature 387, 278-281. doi: $10.1038 / 387278 \mathrm{a} 0$

Brozoski, T. J., Bauer, C. A., and Caspary, D. M. (2002). Elevated fusiform cell activity in the dorsal cochlear nucleus of chinchillas with psychophysical evidence of tinnitus. J. Neurosci. 22, 2383-2390.

Chase, S. M., and Young, E. D. (2007). First-spike latency information in single neurons increases when referenced to population onset. Proc. Natl. Acad. Sci. U.S.A. 104, 5175-5180. doi: 10.1073/pnas.0610368104

Dahmen, J. C., Hartley, D. E., and King, A. J. (2008). Stimulus-timing-dependent plasticity of cortical frequency representation. J. Neurosci. 28, 13629-13639. doi: 10.1523/JNEUROSCI.4429-08.2008

Davis, K. A., Miller, R. L., and Young, E. D. (1996). Effects of somatosensory and parallel-fiber stimulation on neurons in dorsal cochlear nucleus. J. Neurophysiol. 76, 3012-3024.

Davis, K. A., and Young, E. D. (1997). Granule cell activation of complex-spiking neurons in dorsal cochlear nucleus. J. Neurosci. 17, 6798-6806.

Dehmel, S., Pradhan, S., Koehler, S., Bledsoe, S., and Shore, S. (2012). Noise overexposure alters long-term somatosensory-auditory processing in the dorsal cochlear nucleus-possible basis for tinnitus-related hyperactivity? J. Neurosci. 32, 1660-1671. doi: 10.1523/JNEUROSCI.4608-11.2012

Frick, A., Magee, J., and Johnston, D. (2004). LTP is accompanied by an enhanced local excitability of pyramidal neuron dendrites. Nat. Neurosci. 7, 126-135. doi: $10.1038 / \mathrm{nn} 1178$

Fujino, K., and Oertel, D. (2003). Bidirectional synaptic plasticity in the cerebellum-like mammalian dorsal cochlear nucleus. Proc. Natl. Acad. Sci. U.S.A. 100, 265-270. doi: 10.1073/pnas.0135345100

Golding, N. L., and Oertel, D. (1997). Physiological identification of the targets of cartwheel cells in the dorsal cochlear nucleus. J. Neurophysiol. 78, 248-260.

Haenggeli, C. A., Pongstaporn, T., Doucet, J. R., and Ryugo, D. K. (2005). Projections from the spinal trigeminal nucleus to the cochlear nucleus in the rat. J. Comp. Neurol. 484, 191-205. doi: 10.1002/cne.20466

Harvey-Girard, E., Lewis, J., and Maler, L. (2010). Burst-induced anti-Hebbian depression acts through short-term synaptic dynamics to cancel redundant for tinnitus-related pathology beginning at the level of the DCN and are currently being explored in an animal model of tinnitus.

\section{Funding}

This study was supported by the National Institutes of Health Grants R01-DC004825 (SS), T32-DC00011 (CW), and the Wallace H. Coulter Translational Research Partnership.

\section{Author Contributions}

$\mathrm{CW}, \mathrm{DM}$, and SS designed research; CW and DM performed research; CW analyzed data; CW and SS wrote the paper.

\section{Acknowledgments}

We thank James Wiler and Chris Ellinger for assistance in experimental setup and instrumentation, and Sandy Bledsoe for comments and insights.

sensory signals. J. Neurosci. 30, 6152-6169. doi: 10.1523/JNEUROSCI.030310.2010

Johnston, D., Christie, B. R., Frick, A., Gray, R., Hoffman, D. A., Schexnayder, L. K., et al. (2003). Active dendrites, potassium channels and synaptic plasticity. Philos. Trans. R. Soc. Lond. B Biol. Sci. 358, 667-674. doi: 10.1098/rstb.2002.1248

Kaltenbach, J. A., Zacharek, M. A., Zhang, J., and Frederick, S. (2004). Activity in the dorsal cochlear nucleus of hamsters previously tested for tinnitus following intense tone exposure. Neurosci. Lett. 355, 121-125. doi: 10.1016/j.neulet.2003.10.038

Kaltenbach, J. A., Zhang, J., and Finlayson, P. (2005). Tinnitus as a plastic phenomenon and its possible neural underpinnings in the dorsal cochlear nucleus. Hear. Res. 206, 200-226. doi: 10.1016/j.heares.2005.02.013

Kanold, P. O., Davis, K. A., and Young, E. D. (2011). Somatosensory context alters auditory responses in the cochlear nucleus. J. Neurophysiol. 105, 1063-1070. doi: 10.1152/jn.00807.2010

Kanold, P. O., and Manis, P. B. (1999). Transient potassium currents regulate the discharge patterns of dorsal cochlear nucleus pyramidal cells. J. Neurosci. 19, 2195-2208.

Kanold, P. O., and Manis, P. B. (2001). A physiologically based model of discharge pattern regulation by transient $\mathrm{K}+$ currents in cochlear nucleus pyramidal cells. J. Neurophysiol. 85, 523-538.

Kanold, P. O., and Manis, P. B. (2005). Encoding the timing of inhibitory inputs. J. Neurophysiol. 93, 2887-2897. doi: 10.1152/jn.00910.2004

Kanold, P. O., and Young, E. D. (2001). Proprioceptive information from the pinna provides somatosensory input to cat dorsal cochlear nucleus. J. Neurosci. 21, 7848-7858.

Koehler, S. D., Pradhan, S., Manis, P. B., and Shore, S. E. (2011). Somatosensory inputs modify auditory spike timing in dorsal cochlear nucleus principal cells. Eur. J. Neurosci. 33, 409-420. doi: 10.1111/j.1460-9568.2010. 07547.x

Koehler, S. D., and Shore, S. E. (2013a). Stimulus timing-dependent plasticity in dorsal cochlear nucleus is altered in tinnitus. J. Neurosci. 33, 19647-19656. doi: 10.1523/JNEUROSCI.2788-13.2013

Koehler, S. D., and Shore, S. E. (2013b). Stimulus-timing dependent multisensory plasticity in the guinea pig dorsal cochlear nucleus. PLOS ONE 8:e59828. doi: 10.1371/journal.pone.0059828

Manis, P. B. (1990). Membrane properties and discharge characteristics of guinea pig dorsal cochlear nucleus neurons studied in vitro. J. Neurosci. 10, 2338-2351. 
Mu, Y., and Poo, M. M. (2006). Spike timing-dependent LTP/LTD mediates visual experience-dependent plasticity in a developing retinotectal system. Neuron 50, 115-125. doi: 10.1016/j.neuron.2006.03.009

Mugnaini, E., Osen, K. K., Dahl, A. L., Friedrich, V. L. Jr., and Korte, G. (1980). Fine structure of granule cells and related interneurons (termed Golgi cells) in the cochlear nuclear complex of cat, rat and mouse. J. Neurocytol. 9, 537-570. doi: 10.1007/BF01204841

Muniak, M. A., and Ryugo, D. K. (2014). Tonotopic organization of vertical cells in the dorsal cochlear nucleus of the CBA/J mouse. J. Comp. Neurol. 522, 937-949. doi: $10.1002 / \mathrm{cne} .23454$

Nelson, S. B. (2004). Hebb and anti-Hebb meet in the brainstem. Nat. Neurosci. 7, 687-688. doi: 10.1038/nn0704-687

Oertel, D., and Young, E. D. (2004). What's a cerebellar circuit doing in the auditory system? Trends Neurosci. 27, 104-110. doi: 10.1016/j.tins.2003.12.001

Piochon, C., Kruskal, P., Maclean, J., and Hansel, C. (2012). Non-Hebbian spiketiming-dependent plasticity in cerebellar circuits. Front. Neural Circuits 6:124. doi: 10.3389/fncir.2012.00124

Requarth, T., and Sawtell, N. B. (2011). Neural mechanisms for filtering selfgenerated sensory signals in cerebellum-like circuits. Curr. Opin. Neurobiol. 21, 602-608. doi: 10.1016/j.conb.2011.05.031

Rhode, W. S. (1999). Vertical cell responses to sound in cat dorsal cochlear nucleus. J. Neurophysiol. 82, 1019-1032.

Roberts, L. E., Eggermont, J. J., Caspary, D. M., Shore, S. E., Melcher, J. R., and Kaltenbach, J. A. (2010). Ringing ears: the neuroscience of tinnitus. J. Neurosci. 30, 14972-14979. doi: 10.1523/JNEUROSCI.4028-10.2010

Roberts, P. D., and Leen, T. K. (2010). Anti-hebbian spike-timing-dependent plasticity and adaptive sensory processing. Front. Comput. Neurosci. 4:156. doi: $10.3389 /$ fncom.2010.00156

Sedlacek, M., and Brenowitz, S. D. (2014). Cell-type specific short-term plasticity at auditory nerve synapses controls feed-forward inhibition in the dorsal cochlear nucleus. Front. Neural Circuits 8:78. doi: 10.3389/fncir.2014.00078

Sedlacek, M., Tipton, P. W., and Brenowitz, S. D. (2011). Sustained firing of cartwheel cells in the dorsal cochlear nucleus evokes endocannabinoid release and retrograde suppression of parallel fiber synapses. J. Neurosci. 31, 1580715817. doi: 10.1523/JNEUROSCI.4088-11.2011

Shore, S. E. (2005). Multisensory integration in the dorsal cochlear nucleus: unit responses to acoustic and trigeminal ganglion stimulation. Eur. J. Neurosci. 21, 3334-3348. doi: 10.1111/j.1460-9568.2005.04142.x

Shore, S. E., El Kashlan, H., and Lu, J. (2003). Effects of trigeminal ganglion stimulation on unit activity of ventral cochlear nucleus neurons. Neuroscience 119, 1085-1101. doi: 10.1016/S0306-4522(03)00207-0

Shore, S. E., Vass, Z., Wys, N. L., and Altschuler, R. A. (2000). Trigeminal ganglion innervates the auditory brainstem. J. Comp. Neurol. 419, 271-285. doi: 10.1002/(SICI)1096-9861(20000410)419:3<271::AID-CNE1>3.0.CO;2-M

Smith, P. H., and Rhode, W. S. (1985). Electron microscopic features of physiologically characterized, HRP-labeled fusiform cells in the cat dorsal cochlear nucleus. J. Comp. Neurol. 237, 127-143. doi: 10.1002/cne.902370110
Stabler, S. E., Palmer, A. R., and Winter, I. M. (1996). Temporal and mean rate discharge patterns of single units in the dorsal cochlear nucleus of the anesthetized guinea pig. J. Neurophysiol. 76, 1667-1688.

Stefanescu, R. A., Koehler, S. D., and Shore, S. E. (2015). Stimulustiming-dependent modifications of rate-level functions in animals with and without tinnitus. J. Neurophysiol. 113, 956-970. doi: 10.1152/jn.0045 7.2014

Tzounopoulos, T., Kim, Y., Oertel, D., and Trussell, L. O. (2004). Cell-specific, spike timing-dependent plasticities in the dorsal cochlear nucleus. Nat. Neurosci. 7, 719-725. doi: 10.1038/nn1272

Tzounopoulos, T., Rubio, M. E., Keen, J. E., and Trussell, L. O. (2007). Coactivation of pre- and postsynaptic signaling mechanisms determines cell-specific spike-timing-dependent plasticity. Neuron 54, 291-301. doi: 10.1016/j.neuron.2007.03.026

Wright, D. D., and Ryugo, D. K. (1996). Mossy fiber projections from the cuneate nucleus to the cochlear nucleus in the rat. J. Comp. Neurol. 365, 159-172. doi: 10.1002/(SICI)1096-9861(19960129)365:1<159::AID-CNE12> 3.0.CO;2-L

Yao, H., and Dan, Y. (2001). Stimulus timing-dependent plasticity in cortical processing of orientation. Neuron 32, 315-323. doi: 10.1016/S0896-6273(01) 00460-3

Zeng, C., Shroff, H., and Shore, S. E. (2011). Cuneate and spinal trigeminal nucleus projections to the cochlear nucleus are differentially associated with vesicular glutamate transporter-2. Neuroscience 176, 142-151. doi: 10.1016/j.neuroscience.2010.12.010

Zhan, X., Pongstaporn, T., and Ryugo, D. K. (2006). Projections of the second cervical dorsal root ganglion to the cochlear nucleus in rats. J. Comp. Neurol. 496, 335-348. doi: 10.1002/cne.20917

Zhao, Y., and Tzounopoulos, T. (2011). Physiological activation of cholinergic inputs controls associative synaptic plasticity via modulation of endocannabinoid signaling. J. Neurosci. 31, 3158-3168. doi: 10.1523/JNEUROSCI.5303-10.2011

Zhou, J., and Shore, S. (2004). Projections from the trigeminal nuclear complex to the cochlear nuclei: a retrograde and anterograde tracing study in the guinea pig. J. Neurosci. Res. 78, 901-907. doi: 10.1002/jnr. 20343

Conflict of Interest Statement: The authors declare that the research was conducted in the absence of any commercial or financial relationships that could be construed as a potential conflict of interest.

Copyright (c) $2015 \mathrm{Wu}$, Martel and Shore. This is an open-access article distributed under the terms of the Creative Commons Attribution License (CC BY). The use, distribution or reproduction in other forums is permitted, provided the original author(s) or licensor are credited and that the original publication in this journal is cited, in accordance with accepted academic practice. No use, distribution or reproduction is permitted which does not comply with these terms. 